Research Article

\title{
Global Asymptotic Stability for Nonlinear Functional Integral Equation of Mixed Type
}

\author{
Zhinan Xia \\ Department of Applied Mathematics, Zhejiang University of Technology, Hangzhou, Zhejiang 310023, China \\ Correspondence should be addressed to Zhinan Xia; xiazn299@zjut.edu.cn
}

Received 29 May 2013; Revised 27 August 2013; Accepted 27 August 2013

Academic Editor: Naseer Shahzad

Copyright ( 2013 Zhinan Xia. This is an open access article distributed under the Creative Commons Attribution License, which permits unrestricted use, distribution, and reproduction in any medium, provided the original work is properly cited.

The existence results of global asymptotic stability of the solution are proved for functional integral equation of mixed type. The measure of noncompactness and the fixed-point theorem of Darbo are the main tools in carrying out our proof. Furthermore, some examples are given to show the efficiency and usefulness of the main findings.

\section{Introduction}

It is well known that functional integral equation of various types creates an important subject of numerous mathematical investigations and constitutes a significant branch of nonlinear analysis. It has great applications in physics, engineering, economics, and biologyed modelling problems connect with real world. With the help of several tools of functional analysis, topology, and fixed-point theory, many authors have made important contributions to this theory [1-5].

Let $\mathbb{R}$ denote the real line and $\mathbb{R}^{+}=[0,+\infty)$. In this paper, we investigate the nonlinear functional integral equation of mixed type, namely,

$$
\begin{aligned}
x(t)=F(t, & f(t, x(\alpha(t))), \\
& \int_{0}^{\beta(t)} u(t, s, x(\gamma(s))) d_{s} g(t, s), \\
& \left.\int_{0}^{\infty} v(t, s, x(\delta(s))) d s\right), \quad t \in \mathbb{R}^{+} .
\end{aligned}
$$

It is worthwhile mentioning that (1) contains numerous functions and integral and functional integral equations encountered in nonlinear analysis. For example, the classical Volterra integral equation, the famous Chandrasekhar integral equation, the Urysohn integral equation, and the Volterra-Stieltjes integral equation are the special cases of (1).

The goal of this paper is to investigate the existence and asymptotic behavior of solutions for (1). Using the technique associated with a suitable measure of noncompactness and the fixed-point theorem of Darbo, we show that (1) has at least one solution under rather general and convenient assumptions. We also obtain some asymptotic characterization of the solutions of (1). The results of the present paper generalize several ones obtained previously in the papers $[1,3,6-8]$ and references therein.

\section{Preliminaries}

In this section, we introduce some notations, definitions, and preliminary facts which are used in this paper. Let $x$ be a real function defined on $[a, b]$, and let $\bigvee_{a}^{b} x$ denotes the variation of $x$ on $[a, b]$; if $\bigvee_{a}^{b} x$ is finite, we say that $x$ is of bounded variation. Let $g(t, s):[a, b] \times[c, d] \rightarrow \mathbb{R}$ be a function, and let $\bigvee_{t=p}^{q} g(t, s)$ indicate the variation of the function $t \rightarrow$ $g(t, s)$ on $[p, q] \subset[a, b]$, where $s$ is arbitrarily fixed in $[c, d]$. Similarly, define $\bigvee_{s=p}^{q} g(t, s)$. If $x$ and $\varphi$ are two real functions defined on $[a, b]$, define the Stieltjes integral $\int_{a}^{b} x(t) d \varphi(t)$ and say that $x$ is Stieltjes integrable on $[a, b]$ with respect to $\varphi$.

Lemma 1 (see [9]). If $x$ is Stieltjes integrable on $[a, b]$ with respect to $\varphi$ of bounded variation; then

$$
\left|\int_{a}^{b} x(t) d \varphi(t)\right| \leq \int_{a}^{b}|x(t)| d\left(\bigvee_{a}^{t} \varphi\right)
$$


Lemma 2 (see [9]). Let $x_{1}, x_{2}$ be Stieltjes integrable functions on $[a, b]$ with respect to a nondecreasing function $\varphi$ such that $x_{1}(t) \leq x_{2}(t)$ for $t \in[a, b]$, then

$$
\int_{a}^{b} x_{1}(t) d \varphi(t) \leq \int_{a}^{b} x_{2}(t) d \varphi(t) .
$$

We also consider Stieltjes integrals $\int_{a}^{b} x(s) d_{s} g(t, s)$, where $g:[a, b] \times[a, b] \rightarrow \mathbb{R}$ and $d_{s}$ indicates the integration with respect to $s$. The details concerning the integral of this type will be given later.

Let $(E,\|\cdot\|)$ be a real Banach space with zero element $\theta$. Denote by $B(x, r)$ the closed ball centered at $x$ and with radius $r, B_{r}$ stands for the ball $B(\theta, r)$. If $X$ is a nonempty subset of $E$, we denote by $\bar{X}$ and $\operatorname{Conv} X$ the closure and the closed convex hull of $X$, respectively. The family of all nonempty and bounded subsets of $E$ is denoted by $\mathfrak{M}_{E}$ and its subfamily consisting of all relatively compact sets is denoted by $\mathfrak{N}_{E}$.

Definition 3 (see [10]). A function $\mu: \mathfrak{M}_{E} \rightarrow \mathbb{R}^{+}:=[0, \infty)$ is said to be a measure of noncompactness in $E$ if it satisfies the following conditions.

(1) The family $\operatorname{ker} \mu=\left\{X \in \mathfrak{M}_{E}: \mu(X)=0\right\}$ is nonempty and $\operatorname{ker} \mu \subset \mathfrak{N}_{E}$.

(2) $X \subset Y \Rightarrow \mu(X) \leq \mu(Y)$.

(3) $\mu(\bar{X})=\mu(\operatorname{Conv} X)=\mu(X)$.

(4) $\mu(\lambda X+(1-\lambda) Y) \leq \lambda \mu(X)+(1-\lambda) \mu(Y)$ for $\lambda \in[0,1]$.

(5) If $\left\{X_{n}\right\}_{n}$ is a sequence of closed sets from $\mathfrak{M}_{E}$ such that $X_{n+1} \subset X_{n}(n=1,2, \ldots)$ and if $\lim _{n \rightarrow \infty} \mu\left(X_{n}\right)=0$, then the set $X_{\infty}=\bigcap_{n=1}^{\infty} X_{n}$ is nonempty.

The family $\operatorname{ker} \mu$ in (1) is called the kernel of $\mu$. Note that $X_{\infty}$ from (5) is a member of $\operatorname{ker} \mu$.

Consider the Banach space $\mathrm{BC}\left(\mathbb{R}^{+}\right)$of all real functions defined, bounded and continuous on $\mathbb{R}^{+}$, and equipped with the maximum norm $\|x\|=\sup \{|x(t)|: t \geq 0\}$. Fix a nonempty bounded subset $X$ of $\mathrm{BC}\left(\mathbb{R}^{+}\right)$and $T>0$, and for $x \in X, \varepsilon>0$, define

$$
\omega^{T}(x, \varepsilon):=\sup \{|x(t)-x(s)|: t, s \in[0, T],|t-s| \leq \varepsilon\} .
$$

Let

$$
\begin{gathered}
\omega^{T}(X, \varepsilon)=\sup \left\{\omega^{T}(x, \varepsilon): x \in X\right\}, \\
\omega_{0}^{T}(X)=\lim _{\varepsilon \rightarrow 0} \omega^{T}(X, \varepsilon), \\
\omega_{0}(X)=\lim _{T \rightarrow \infty} \omega_{0}^{T}(X) .
\end{gathered}
$$

Moreover, for a fixed $t \in \mathbb{R}^{+}$, define

$$
\begin{gathered}
X(t)=\{x(t): x \in X\}, \\
\operatorname{diam} X(t)=\sup \{|x(t)-y(t)|: x, y \in X\} .
\end{gathered}
$$

Finally, define the function $\mu$ on $\mathfrak{M}_{\mathrm{BC}\left(\mathbb{R}^{+}\right)}$by

$$
\mu(X)=\omega_{0}(X)+\limsup _{t \rightarrow \infty} \operatorname{diam} X(t) .
$$

It can be shown that $\mu$ is a measure of noncompactness in $\mathrm{BC}\left(\mathbb{R}^{+}\right)[10]$.

Remark 4. ker $\mu$ contains nonempty and bounded sets $X$ such that functions from $X$ are locally equicontinuous on $\mathbb{R}^{+}$and tend to zero at infinity uniformly with respect to $X$.

Assume $\Omega$ is a nonempty subset of $\mathrm{BC}\left(\mathbb{R}^{+}\right)$and $Q$ is an operator defined on $\Omega$ with values in $\mathrm{BC}\left(\mathbb{R}^{+}\right)$. Consider the operator equation

$$
x(t)=(Q x)(t), \quad t \in \mathbb{R}^{+} .
$$

Definition 5 (see [8]). The solution $x(t)$ of (8) is said to be locally attractive if there exists a ball $B\left(x_{0}, r\right)$ in $\mathrm{BC}\left(\mathbb{R}^{+}\right)$such that for arbitrary solutions $x=x(t)$ and $y=y(t)$ of $(8)$ belonging to $B\left(x_{0}, r\right) \cap \Omega$ such that

$$
\lim _{t \rightarrow \infty}(x(t)-y(t))=0 .
$$

Definition 6 (see [3]). The solution $x=x(t)$ of (8) is said to be globally attractive, if (9) holds for each solutions $y=y(t)$ of (8). If (9) is satisfied uniformly with respect to $\Omega$, we say that solutions of (8) are globally asymptotically stable (or uniformly globally attractive).

Theorem 7 (Darbo fixed-point theorem [10]). Let $\Omega$ be a nonempty bounded closed and convex subset of Banach space $E$ and let $Q: \Omega \rightarrow \Omega$ be a continuous mapping such that $\mu(Q X) \leq k \mu(X)$ for any nonempty subset of $X$ of $\Omega$, where $k \in[0,1)$ is a constant. Then $Q$ has a fixed-point in $\Omega$.

From Theorem 7, it can be shown that the set fix $Q$ of fixed-points of $Q$ is a member of $\operatorname{ker} \mu$.

\section{Main Results}

In this section, we will investigate the existence and asymptotic behavior of solutions for $(1)$ in $\mathrm{BC}\left(\mathbb{R}^{+}\right)$. Assume that the following conditions are satisfied.

$\left(H_{1}\right) \alpha, \beta, \gamma, \delta: \mathbb{R}^{+} \rightarrow \mathbb{R}^{+}$are continuous functions.

$\left(H_{2}\right) g: \mathbb{R}^{+} \times \mathbb{R}^{+} \rightarrow \mathbb{R}$ satisfies the following conditions.

$\left(H_{2 . a}\right)$ For all $t_{1}, t_{2} \in \mathbb{R}^{+}$such that $t_{1}<t_{2}$, the function $s \rightarrow g\left(t_{2}, s\right)-g\left(t_{1}, s\right)$ is nondecreasing on $\mathbb{R}^{+}$.

$\left(H_{2 . b}\right)$ The function $s \rightarrow g(0, s)$ is nondecreasing on $\mathbb{R}^{+}$.

$\left(H_{2 . c}\right)$ The functions $s \rightarrow g(t, s)$ and $t \rightarrow g(t, s)$ are continuous on $\mathbb{R}^{+}$for each fixed $t \in \mathbb{R}^{+}$or $s \in$ $\mathbb{R}^{+}$, respectively.

$\left(H_{3}\right) u: \mathbb{R}^{+} \times \mathbb{R}^{+} \times \mathbb{R} \rightarrow \mathbb{R}$ is a continuous function and there exist a continuous function $k(t, s): \mathbb{R}^{+} \times \mathbb{R}^{+} \rightarrow$ $\mathbb{R}^{+}$and a continuous, nondecreasing function $\varphi$ : $\mathbb{R}^{+} \rightarrow \mathbb{R}^{+}$such that

$$
|u(t, s, x)| \leq k(t, s) \varphi(|x|), \quad \forall t, s \in \mathbb{R}^{+}, x \in \mathbb{R} .
$$


$\left(H_{4}\right) v: \mathbb{R}^{+} \times \mathbb{R}^{+} \times \mathbb{R} \rightarrow \mathbb{R}$ is a continuous function, $v(t, s, 0)=0$ and there exist a continuous function $p(t, s): \mathbb{R}^{+} \times \mathbb{R}^{+} \rightarrow \mathbb{R}^{+}$and a continuous, nondecreasing functions $\psi: \mathbb{R}^{+} \rightarrow \mathbb{R}^{+}$such that $\psi(0)=$ 0 and

$$
\begin{aligned}
& |v(t, s, x)-v(t, s, y)| \\
& \quad \leq p(t, s) \psi(|x-y|), \quad \forall t, s \in \mathbb{R}^{+}, x, y \in \mathbb{R} .
\end{aligned}
$$

$\left(H_{5}\right) F: \mathbb{R}^{+} \times \mathbb{R} \times \mathbb{R} \times \mathbb{R} \rightarrow \mathbb{R}$ is a continuous function and there exist a constant $M>0$ and continuous functions $m_{1}(t), m_{2}(t): \mathbb{R}^{+} \rightarrow \mathbb{R}^{+}$such that

$$
\begin{aligned}
&\left|F\left(t, x_{1}, x_{2}, x_{3}\right)-F\left(t, y_{1}, y_{2}, y_{3}\right)\right| \\
& \leq M\left|x_{1}-y_{1}\right|+m_{1}(t)\left|x_{2}-y_{2}\right| \\
&+m_{2}(t)\left|x_{3}-y_{3}\right|,
\end{aligned}
$$

for all $t \in \mathbb{R}^{+}, x_{1}, x_{2}, x_{3}, y_{1}, y_{2}, y_{3} \in \mathbb{R}$. Moreover, the function $t \rightarrow F(t, 0,0,0)$ is bounded with $F_{0}=$ $\sup _{t \geq 0}|F(t, 0,0,0)|$.

$\left(H_{6}\right) f: \mathbb{R}^{+} \times \mathbb{R} \rightarrow \mathbb{R}$ is a continuous function and there exists a constant $\lambda>0$ such that

$|f(t, x)-f(t, y)| \leq \lambda|x-y|, \quad \forall x, y \in \mathbb{R}, t \in \mathbb{R}^{+}$.

Moreover, $f(t, 0) \in \mathrm{BC}\left(\mathbb{R}^{+}\right)$and $f_{0}=\sup _{t \geq 0}|f(t, 0)|$.

$\left(H_{7}\right) \lim _{t \rightarrow \infty} m_{1}(t) \int_{0}^{\beta(t)} k(t, s) d_{s} g(t, s) \quad=$ $\lim _{t \rightarrow \infty} m_{2}(t) \int_{0}^{\infty} p(t, s) d s=0$.

$\left(H_{8}\right)$ Consider

$$
\lim _{T \rightarrow \infty}\left\{\sup \left\{\int_{T}^{\infty} p(t, s) d s: t \in \mathbb{R}^{+}\right\}\right\}=0 .
$$

$\left(H_{9}\right)$ There exists a positive solution $r_{0}$ of the inequality

$$
M \lambda r+M f_{0}+M_{1} \varphi(r)+M_{2} \psi(r)+F_{0} \leq r
$$

such that $M \lambda<1$, where

$$
\begin{gathered}
M_{1}=\sup \left\{m_{1}(t) \int_{0}^{\beta(t)} k(t, s) d_{s} g(t, s): t \geq 0\right\}, \\
M_{2}=\sup \left\{m_{2}(t) \int_{0}^{\infty} p(t, s) d s: t \geq 0\right\} .
\end{gathered}
$$

Remark 8 . There are many examples of function $g(t, s)$ which verify $\left(\mathrm{H}_{2}\right)$, such as

(i) $g(t, s)=s$,

(ii) $g(t, s)=s \ln (t+1)$,

(iii) $g(t, s)= \begin{cases}t \ln ((t+s) / t), & \text { for } t>0, s \geq 0 \\ 0, & \text { for } t=0, s \geq 0,\end{cases}$

(iv) $g(t, s)=\int_{0}^{t}\left(\int_{0}^{s} p(z, y) d y\right) d z$, where $p: \mathbb{R}^{+} \times \mathbb{R}^{+} \rightarrow$ $\mathbb{R}^{+}$is a bounded and integrable function.

One can see [2] for more details.
In what follows, we provide some properties of the function $g(t, s)$.

Lemma 9 (see [9]). Under assumptions $\left(H_{2 . a}\right)$ and $\left(H_{2 . b}\right)$, the function $s \rightarrow g(t, s)$ is nondecreasing on $\mathbb{R}^{+}$for any fixed $t \in$ $\mathbb{R}^{+}$.

Lemma 10 (see [9]). Assume that the function g satisfies the assumption $\left(H_{2 . a}\right)$; then for arbitrarily fixed $s_{1}, s_{2} \in \mathbb{R}^{+}$such that $s_{1}<s_{2}$, the function $t \rightarrow g\left(t, s_{2}\right)-g\left(t, s_{1}\right)$ is nondecreasing on $\mathbb{R}^{+}$.

Remark 11. Observe that if $r_{0}>0$ satisfies (15), then

$$
M \lambda \leq 1-\frac{M f_{0}}{r_{0}}-\frac{F_{0}}{r_{0}}-\frac{M_{1} \varphi\left(r_{0}\right)}{r_{0}}-\frac{M_{2} \psi\left(r_{0}\right)}{r_{0}} .
$$

Thus, if one of the terms of $M f_{0}, F_{0}, M_{1}$, and $M_{2}$ dose not vanish, then $M \lambda<1$ is automatically satisfied.

For the sake of convenience and simplicity, we will use the following notations in the paper. For fixed $T>0$, let

$$
\begin{aligned}
& \alpha^{T}=\sup \{\alpha(t): t \in[0, T]\}, \\
& \beta^{T}=\sup \{\beta(t): t \in[0, T]\}, \\
& m_{1 T}=\inf \left\{m_{1}(t): t \in[0, T]\right\}, \\
& m_{2 T}=\inf \left\{m_{2}(t): t \in[0, T]\right\}, \\
& m_{1}^{T}=\sup \left\{m_{1}(t): t \in[0, T]\right\}, \\
& k_{T}=\sup \left\{k(t, s): t \in[0, T], s \in\left[0, \beta^{T}\right]\right\} .
\end{aligned}
$$

The main result of this section is the following theorem.

Theorem 12. Under assumptions $\left(H_{1}\right)-\left(H_{9}\right)$, (1) has at least one solution $x \in B C\left(\mathbb{R}^{+}\right)$. Moreover, solutions of (1) are globally asymptotically stable.

Proof. Consider the operator $\mathscr{F}$ defined on $\mathrm{BC}\left(\mathbb{R}^{+}\right)$by

$$
\begin{aligned}
(\mathscr{F} x)(t)=F(t, f(t, x(\alpha(t))), & \\
& \int_{0}^{\beta(t)} u(t, s, x(\gamma(s))) d_{s} g(t, s), \\
& \left.\int_{0}^{\infty} v(t, s, x(\delta(s))) d s\right), \quad t \in \mathbb{R}^{+} .
\end{aligned}
$$

Taking into account $\left(\mathrm{H}_{2}\right),\left(\mathrm{H}_{3}\right)$, and Lemma 9, we deduce that $\mathscr{F}$ is well defined on $\mathrm{BC}\left(\mathbb{R}^{+}\right)$. 
First, we show that $\mathscr{F}$ maps $\mathrm{BC}\left(\mathbb{R}^{+}\right)$into itself. It is clear that $\mathscr{F} x$ is continuous on $\mathbb{R}^{+}$for each $x \in \mathrm{BC}\left(\mathbb{R}^{+}\right)$. By $\left(H_{3}\right)-\left(H_{6}\right)$ and Lemma 1 , for $t \in \mathbb{R}^{+}$, one has

$$
\begin{aligned}
& |(\mathscr{F} x)(t)| \\
& \leq \mid F(t, f(t, x(\alpha(t))), \\
& \int_{0}^{\beta(t)} u(t, s, x(\gamma(s))) d_{s} g(t, s), \\
& \left.\int_{0}^{\infty} v(t, s, x(\delta(s))) d s\right) \\
& \leq \mid F(t, f(t, x(\alpha(t))), \\
& \int_{0}^{\beta(t)} u(t, s, x(\gamma(s))) d_{s} g(t, s), \\
& \left.\int_{0}^{\infty} v(t, s, x(\delta(s))) d s\right)-F(t, 0,0,0) \\
& +F(t, 0,0,0) \\
& \leq M|f(t, x(\alpha(t)))|+m_{1}(t) \\
& \times\left|\int_{0}^{\beta(t)} u(t, s, x(\gamma(s))) d_{s} g(t, s)\right| \\
& +m_{2}(t)\left|\int_{0}^{\infty} v(t, s, x(\delta(s))) d s\right|+|F(t, 0,0,0)| \\
& \leq M \lambda|x(\alpha(t))|+M|f(t, 0)|+m_{1}(t) \\
& \times \int_{0}^{\beta(t)}|u(t, s, x(\gamma(s)))| d_{s}\left(\bigvee_{p=0}^{s} g(t, p)\right) \\
& +m_{2}(t) \int_{0}^{\infty}|v(t, s, x(\delta(s)))| d s+|F(t, 0,0,0)| \\
& \leq M \lambda\|x\|+M|f(t, 0)|+m_{1}(t) \\
& \times \int_{0}^{\beta(t)} k(t, s) \varphi(|x(\gamma(s))|) d_{s} g(t, s) \\
& +m_{2}(t) \int_{0}^{\infty} p(t, s) \psi(|x(\delta(s))|) d s+|F(t, 0,0,0)| \\
& \leq M \lambda\|x\|+M f_{0}+\varphi(\|x\|) m_{1}(t) \\
& \times \int_{0}^{\beta(t)} k(t, s) d_{s} g(t, s) \\
& +\psi(\|x\|) m_{2}(t) \int_{0}^{\infty} p(t, s) d s+F_{0} \\
& \leq M \lambda\|x\|+M f_{0}+M_{1} \varphi(\|x\|) \\
& +M_{2} \psi(\|x\|)+F_{0},
\end{aligned}
$$

By $\left(H_{9}\right)$, it is clear that $\mathscr{F}\left(B_{r_{0}}\right) \subset B_{r_{0}}$.
Next, we show that $\mathscr{F}$ is continuous on $B_{r_{0}}$. Let $\varepsilon>0$ be given, and since $\left(H_{7}\right)$ holds, there exists $T>0$, such that $m_{1}(t) \int_{0}^{\beta(t)} k(t, s) d_{s} g(t, s)<\varepsilon$ and $m_{2}(t) \int_{0}^{\infty} p(t, s) d s<\varepsilon$ for $t \geq T$. Consider the following two cases.

Case 1. For $t \geq T, x, y \in B_{r_{0}}$ with $\|x-y\| \leq \varepsilon$,

$$
\begin{aligned}
& |(\mathscr{F} x)(t)-(\mathscr{F} y)(t)| \\
& \leq \mid F(t, f(t, x(\alpha(t))), \\
& \int_{0}^{\beta(t)} u(t, s, x(\gamma(s))) d_{s} g(t, s), \\
& \left.\int_{0}^{\infty} v(t, s, x(\delta(s))) d s\right) \\
& -F(t, f(t, y(\alpha(t))), \\
& \int_{0}^{\beta(t)} u(t, s, y(\gamma(s))) d_{s} g(t, s), \\
& \left.\int_{0}^{\infty} v(t, s, y(\delta(s))) d s\right) \\
& \leq M|f(t, x(\alpha(t)))-f(t, y(\alpha(t)))|+m_{1}(t) \\
& \times \mid \int_{0}^{\beta(t)}[u(t, s, x(\gamma(s))) \\
& -u(t, s, y(\gamma(s)))] d_{s} g(t, s) \\
& +m_{2}(t)\left|\int_{0}^{\infty}[v(t, s, x(\delta(s)))-v(t, s, y(\delta(s)))] d s\right| \\
& \leq M \lambda|x(\alpha(t))-y(\alpha(t))|+m_{1}(t) \\
& \times \int_{0}^{\beta(t)}|u(t, s, x(\gamma(s)))-u(t, s, y(\gamma(s)))| d_{s} \\
& \times\left(\bigvee_{p=0}^{s} g(t, p)\right)+m_{2}(t) \\
& \times \int_{0}^{\infty}|v(t, s, x(\delta(s)))-v(t, s, y(\delta(s)))| d s \\
& \leq M \lambda\|x-y\|+m_{1}(t) \\
& \times \int_{0}^{\beta(t)}|u(t, s, x(\gamma(s)))-u(t, s, y(\gamma(s)))| d_{s} g(t, s) \\
& +m_{2}(t) \int_{0}^{\infty}|v(t, s, x(\delta(s)))| d s \\
& +m_{2}(t) \int_{0}^{\infty}|v(t, s, y(\delta(s)))| d s
\end{aligned}
$$




$$
\begin{aligned}
& \leq M \lambda\|x-y\|+m_{1}(t) \\
& \times \int_{0}^{\beta(t)} k(t, s) \varphi(|x(\gamma(s))|) d_{s} g(t, s) \\
& +m_{1}(t) \int_{0}^{\beta(t)} k(t, s) \varphi(|y(\gamma(s))|) d_{s} g(t, s) \\
& +m_{2}(t) \int_{0}^{\infty} p(t, s) \psi(|x(\delta(s))|) d s \\
& +m_{2}(t) \int_{0}^{\infty} p(t, s) \psi(|y(\delta(s))|) d s \\
& \leq M \lambda\|x-y\|+\varphi(\|x\|) m_{1}(t) \\
& \times \int_{0}^{\beta(t)} k(t, s) d_{s} g(t, s)+\varphi(\|y\|) m_{1}(t) \\
& \times \int_{0}^{\beta(t)} k(t, s) d_{s} g(t, s) \\
& +\psi(\|x\|) m_{2}(t) \int_{0}^{\infty} p(t, s) d s \\
& +\psi(\|y\|) m_{2}(t) \int_{0}^{\infty} p(t, s) d s \\
& \leq M \lambda\|x-y\|+2 \varphi\left(r_{0}\right) m_{1}(t) \\
& \times \int_{0}^{\beta(t)} k(t, s) d_{s} g(t, s) \\
& +2 \psi\left(r_{0}\right) m_{2}(t) \int_{0}^{\infty} p(t, s) d s \\
& \leq M \lambda \varepsilon+2 \varphi\left(r_{0}\right) \varepsilon+2 \psi\left(r_{0}\right) \varepsilon \text {. }
\end{aligned}
$$

Case 2. For $t \in[0, T], x, y \in B_{r_{0}}$ with $\|x-y\| \leq \varepsilon$, by Lemmas 9 and 10 , one has

$$
\begin{aligned}
\mid(\mathscr{F} x) & (t)-(\mathscr{F} y)(t) \mid \\
\leq & M|f(t, x(\alpha(t)))-f(t, y(\alpha(t)))|+m_{1}(t) \\
\times & \mid \int_{0}^{\beta(t)}[u(t, s, x(\gamma(s))) \\
\times & -u(t, s, y(\gamma(s)))] d_{s} g(t, s) \mid+m_{2}(t) \\
\leq & M \lambda|x(\alpha(t))-y(\alpha(t))|+m_{1}(t) \\
\times & \int_{0}^{\beta(t)}|u(t, s, x(\gamma(s)))-u(t, s, y(\gamma(s)))| d_{s} \\
& \times\left(\bigvee_{p=0}^{s} g(t, p)\right)+m_{2}(t) \\
& \times \int_{0}^{\infty}|v(t, s, x(\delta(s)))-v(t, s, y(\delta(s)))| d s
\end{aligned}
$$

$$
\begin{aligned}
\leq & M \lambda\|x-y\|+m_{1}(t) \\
& \times \int_{0}^{\beta(t)}|u(t, s, x(\gamma(s)))-u(t, s, y(\gamma(s)))| d_{s} g(t, s) \\
& +m_{2}(t) \int_{0}^{\infty} p(t, s) \psi(|x(\delta(s))-y(\delta(s))|) d s \\
\leq & M \lambda \varepsilon+m_{1}^{T} \omega(u, \varepsilon) \int_{0}^{\beta(t)} d_{s} g(t, s) \\
& +\psi(\|x-y\|) m_{2}(t) \int_{0}^{\infty} p(t, s) d s \\
\leq & M \lambda \varepsilon+m_{1}^{T} \omega(u, \varepsilon) \\
& \times(g(t, \beta(t))-g(t, 0))+M_{2} \psi(\varepsilon) \\
\leq & M \lambda \varepsilon+m_{1}^{T} \omega(u, \varepsilon) \\
& \times(g(T, \beta(t))-g(T, 0))+M_{2} \psi(\varepsilon) \\
\leq & M \lambda \varepsilon+m_{1}^{T} \omega(u, \varepsilon) \\
& \times\left(g\left(T, \beta^{T}\right)-g(T, 0)\right)+M_{2} \psi(\varepsilon)
\end{aligned}
$$

where

$$
\begin{gathered}
\omega(u, \varepsilon)=\sup \{|u(t, s, x)-u(t, s, y)|: t \in[0, T], \\
s \in\left[0, \beta^{T}\right], x, y \in\left[-r_{0}, r_{0}\right], \\
\|x-y\| \leq \varepsilon\} .
\end{gathered}
$$

By (21) and (22),

$$
\begin{aligned}
&|(\mathscr{F} x)(t)-(\mathscr{F} y)(t)| \\
& \leq \max \{ M \lambda \varepsilon+2 \varphi\left(r_{0}\right) \varepsilon+2 \psi\left(r_{0}\right) \varepsilon, M \lambda \varepsilon+m_{1}^{T} \omega(u, \varepsilon) \\
&\left.\times\left(g\left(T, \beta^{T}\right)-g(T, 0)\right)+M_{2} \psi(\varepsilon)\right\} .
\end{aligned}
$$

Note that $\omega(u, \varepsilon) \rightarrow 0, \psi(\varepsilon) \rightarrow 0$ as $\varepsilon \rightarrow 0$, and $\mathscr{F}$ is continuous on $B_{r_{0}}$ by (24).

Now, and let $X$ be a nonempty set of $B_{r_{0}}$ and $x \in X$, fix arbitrarily $T>0$ and $\varepsilon>0$, let $t_{1}, t_{2} \in[0, T]$ such that $t_{1}<t_{2}$ and $t_{2}-t_{1} \leq \varepsilon$,

$$
\begin{gathered}
\left|(\mathscr{F} x)\left(t_{2}\right)-(\mathscr{F} x)\left(t_{1}\right)\right| \\
\leq \mid F\left(t_{2}, f\left(t_{2}, x\left(\alpha\left(t_{2}\right)\right)\right),\right. \\
\quad \int_{0}^{\beta\left(t_{2}\right)} u\left(t_{2}, s, x(\gamma(s))\right) d_{s} g\left(t_{2}, s\right), \\
\left.\quad \int_{0}^{\infty} v\left(t_{2}, s, x(\delta(s))\right) d s\right) \\
-F\left(t_{1}, f\left(t_{1}, x\left(\alpha\left(t_{1}\right)\right)\right),\right. \\
\quad \int_{0}^{\beta\left(t_{1}\right)} u\left(t_{1}, s, x(\gamma(s))\right) d_{s} g\left(t_{1}, s\right), \\
\left.\quad \int_{0}^{\infty} v\left(t_{1}, s, x(\delta(s))\right) d s\right) \mid
\end{gathered}
$$




$$
\begin{aligned}
& \leq \mid F\left(t_{2}, f\left(t_{2}, x\left(\alpha\left(t_{2}\right)\right)\right),\right. \\
& \int_{0}^{\beta\left(t_{2}\right)} u\left(t_{2}, s, x(\gamma(s))\right) d_{s} g\left(t_{2}, s\right), \\
& \left.\int_{0}^{\infty} v\left(t_{2}, s, x(\delta(s))\right) d s\right) \\
& -F\left(t_{2}, f\left(t_{1}, x\left(\alpha\left(t_{1}\right)\right)\right),\right. \\
& \int_{0}^{\beta\left(t_{2}\right)} u\left(t_{2}, s, x(\gamma(s))\right) d_{s} g\left(t_{2}, s\right), \\
& \left.\int_{0}^{\infty} v\left(t_{2}, s, x(\delta(s))\right) d s\right) \mid \\
& +\mid F\left(t_{2}, f\left(t_{1}, x\left(\alpha\left(t_{1}\right)\right)\right),\right. \\
& \int_{0}^{\beta\left(t_{2}\right)} u\left(t_{2}, s, x(\gamma(s))\right) d_{s} g\left(t_{2}, s\right), \\
& \left.\int_{0}^{\infty} v\left(t_{2}, s, x(\delta(s))\right) d s\right) \\
& -F\left(t_{2}, f\left(t_{1}, x\left(\alpha\left(t_{1}\right)\right)\right),\right. \\
& \int_{0}^{\beta\left(t_{1}\right)} u\left(t_{1}, s, x(\gamma(s))\right) d_{s} g\left(t_{1}, s\right), \\
& \left.\int_{0}^{\infty} v\left(t_{2}, s, x(\delta(s))\right) d s\right) \\
& +\mid F\left(t_{2}, f\left(t_{1}, x\left(\alpha\left(t_{1}\right)\right)\right),\right. \\
& \int_{0}^{\beta\left(t_{1}\right)} u\left(t_{1}, s, x(\gamma(s))\right) d_{s} g\left(t_{1}, s\right), \\
& \left.\int_{0}^{\infty} v\left(t_{2}, s, x(\delta(s))\right) d s\right) \\
& -F\left(t_{2}, f\left(t_{1}, x\left(\alpha\left(t_{1}\right)\right)\right)\right. \\
& \int_{0}^{\beta\left(t_{1}\right)} u\left(t_{1}, s, x(\gamma(s))\right) d_{s} g\left(t_{1}, s\right), \\
& \left.\int_{0}^{\infty} v\left(t_{1}, s, x(\delta(s))\right) d s\right) \\
& +\mid F\left(t_{2}, f\left(t_{1}, x\left(\alpha\left(t_{1}\right)\right)\right),\right. \\
& \int_{0}^{\beta\left(t_{1}\right)} u\left(t_{1}, s, x(\gamma(s))\right) d_{s} g\left(t_{1}, s\right), \\
& \left.\int_{0}^{\infty} v\left(t_{1}, s, x(\delta(s))\right) d s\right)
\end{aligned}
$$

$$
-F\left(t_{1}, f\left(t_{1}, x\left(\alpha\left(t_{1}\right)\right)\right),\right.
$$$$
\int_{0}^{\beta\left(t_{1}\right)} u\left(t_{1}, s, x(\gamma(s))\right) d_{s} g\left(t_{1}, s\right),
$$$$
\left.\int_{0}^{\infty} v\left(t_{1}, s, x(\delta(s))\right) d s\right)
$$$$
\leq M\left|f\left(t_{2}, x\left(\alpha\left(t_{2}\right)\right)\right)-f\left(t_{1}, x\left(\alpha\left(t_{1}\right)\right)\right)\right|
$$$$
+m_{1}^{T} \mid \int_{0}^{\beta\left(t_{2}\right)} u\left(t_{2}, s, x(\gamma(s))\right) d_{s} g\left(t_{2}, s\right)
$$$$
-\int_{0}^{\beta\left(t_{1}\right)} u\left(t_{1}, s, x(\gamma(s))\right) d_{s} g\left(t_{1}, s\right)
$$$$
+m_{2}^{T} \mid \int_{0}^{\infty} v\left(t_{2}, s, x(\delta(s))\right) d s
$$$$
-\int_{0}^{\infty} v\left(t_{1}, s, x(\delta(s))\right) d s \mid+\omega_{r_{0}}^{T}(F, \varepsilon)
$$$$
:=M \vartheta_{0}+m_{1}^{T} \vartheta_{1}+m_{2}^{T} \vartheta_{2}+\omega_{r_{0}}^{T}(F, \varepsilon),
$$

where

$$
\begin{array}{r}
\vartheta_{0}=\left|f\left(t_{2}, x\left(\alpha\left(t_{2}\right)\right)\right)-f\left(t_{1}, x\left(\alpha\left(t_{1}\right)\right)\right)\right|, \\
\vartheta_{1}=\mid \int_{0}^{\beta\left(t_{2}\right)} u\left(t_{2}, s, x(\gamma(s))\right) d_{s} g\left(t_{2}, s\right) \\
\quad-\int_{0}^{\beta\left(t_{1}\right)} u\left(t_{1}, s, x(\gamma(s))\right) d_{s} g\left(t_{1}, s\right) \mid, \\
\vartheta_{2}=\mid \int_{0}^{\infty} v\left(t_{2}, s, x(\delta(s))\right) d s \\
\quad-\int_{0}^{\infty} v\left(t_{1}, s, x(\delta(s))\right) d s \mid
\end{array}
$$

$\omega_{r_{0}}^{T}(F, \varepsilon)$

$$
\begin{gathered}
=\sup \left\{\left|F\left(t_{2}, x, y, z\right)-F\left(t_{1}, x, y, z\right)\right|\right. \\
: t_{1}, t_{2} \in[0, T],\left|t_{2}-t_{1}\right| \leq \varepsilon, \\
x \in\left[-\lambda r_{0}-f_{0}, \lambda r_{0}+f_{0}\right], \\
y \in\left[-\frac{\varphi\left(r_{0}\right) M_{1}}{m_{1 T}}, \frac{\varphi\left(r_{0}\right) M_{1}}{m_{1 T}}\right], \\
\left.z \in\left[-\frac{M_{2} \psi\left(r_{0}\right)}{m_{2 T}}, \frac{M_{2} \psi\left(r_{0}\right)}{m_{2 T}}\right]\right\} .
\end{gathered}
$$

Moreover,

$$
\begin{aligned}
\vartheta_{0}= & \left|f\left(t_{2}, x\left(\alpha\left(t_{2}\right)\right)\right)-f\left(t_{1}, x\left(\alpha\left(t_{1}\right)\right)\right)\right| \\
\leq & \left|f\left(t_{2}, x\left(\alpha\left(t_{2}\right)\right)\right)-f\left(t_{2}, x\left(\alpha\left(t_{1}\right)\right)\right)\right| \\
& +\left|f\left(t_{2}, x\left(\alpha\left(t_{1}\right)\right)\right)-f\left(t_{1}, x\left(\alpha\left(t_{1}\right)\right)\right)\right|
\end{aligned}
$$




$$
\begin{aligned}
\leq & \lambda\left|x\left(\alpha\left(t_{2}\right)\right)-x\left(\alpha\left(t_{1}\right)\right)\right| \\
& +\left|f\left(t_{2}, x\left(\alpha\left(t_{1}\right)\right)\right)-f\left(t_{1}, x\left(\alpha\left(t_{1}\right)\right)\right)\right| \\
\leq & \lambda \omega^{T}\left(x, \omega^{T}(\alpha, \varepsilon)\right)+\omega_{r_{0}}^{T}(f, \varepsilon),
\end{aligned}
$$

where

$$
\begin{aligned}
& \omega^{T}\left(x, \omega^{T}(\alpha, \varepsilon)\right) \\
& =\sup \left\{\left|x\left(\theta_{2}\right)-x\left(\theta_{1}\right)\right|: \theta_{1}, \theta_{2} \in\left[0, \alpha^{T}\right],\right. \\
& \left.\quad\left|\theta_{2}-\theta_{1}\right|<\omega^{T}(\alpha, \varepsilon)\right\}, \\
& \omega_{r_{0}}^{T}(f, \varepsilon) \\
& =\sup \left\{\left|f\left(t_{2}, x\right)-f\left(t_{1}, x\right)\right|: t_{1}, t_{2} \in[0, T],\right. \\
& \left.\left|t_{2}-t_{1}\right| \leq \varepsilon, x \in\left[-r_{0}, r_{0}\right]\right\} .
\end{aligned}
$$

By Lemma 1, one has

$$
\begin{aligned}
& \vartheta_{1}=\mid \int_{0}^{\beta\left(t_{2}\right)} u\left(t_{2}, s, x(\gamma(s))\right) d_{s} g\left(t_{2}, s\right) \\
& -\int_{0}^{\beta\left(t_{1}\right)} u\left(t_{1}, s, x(\gamma(s))\right) d_{s} g\left(t_{1}, s\right) \\
& \leq \mid \int_{0}^{\beta\left(t_{2}\right)} u\left(t_{2}, s, x(\gamma(s))\right) d_{s} g\left(t_{2}, s\right) \\
& -\int_{0}^{\beta\left(t_{1}\right)} u\left(t_{2}, s, x(\gamma(s))\right) d_{s} g\left(t_{2}, s\right) \\
& +\mid \int_{0}^{\beta\left(t_{1}\right)} u\left(t_{2}, s, x(\gamma(s))\right) d_{s} g\left(t_{2}, s\right) \\
& -\int_{0}^{\beta\left(t_{1}\right)} u\left(t_{1}, s, x(\gamma(s))\right) d_{s} g\left(t_{2}, s\right) \mid \\
& +\mid \int_{0}^{\beta\left(t_{1}\right)} u\left(t_{1}, s, x(\gamma(s))\right) d_{s} g\left(t_{2}, s\right) \\
& -\int_{0}^{\beta\left(t_{1}\right)} u\left(t_{1}, s, x(\gamma(s))\right) d_{s} g\left(t_{1}, s\right) \\
& \leq \int_{\beta\left(t_{1}\right)}^{\beta\left(t_{2}\right)}\left|u\left(t_{2}, s, x(\gamma(s))\right)\right| d_{s} \\
& \times\left(\bigvee_{p=\beta\left(t_{1}\right)}^{s} g\left(t_{2}, p\right)\right) \\
& +\int_{0}^{\beta\left(t_{1}\right)}\left|u\left(t_{2}, s, x(\gamma(s))\right)-u\left(t_{1}, s, x(\gamma(s))\right)\right| d_{s} \\
& \times\left(\bigvee_{p=0}^{s} g\left(t_{2}, p\right)\right) \\
& +\int_{0}^{\beta\left(t_{1}\right)}\left|u\left(t_{1}, s, x(\gamma(s))\right)\right| d_{s} \\
& \times\left(\bigvee_{p=0}^{s}\left[g\left(t_{2}, p\right)-g\left(t_{1}, p\right)\right]\right)
\end{aligned}
$$

$$
\begin{aligned}
\leq & \int_{\beta\left(t_{1}\right)}^{\beta\left(t_{2}\right)}\left|u\left(t_{2}, s, x(\gamma(s))\right)\right| d_{s} g\left(t_{2}, s\right) \\
& +\int_{0}^{\beta\left(t_{1}\right)} \mid u\left(t_{2}, s, x(\gamma(s))\right)
\end{aligned}
$$$$
-u\left(t_{1}, s, x(\gamma(s))\right) \mid d_{s} g\left(t_{2}, s\right)
$$$$
+\int_{0}^{\beta\left(t_{1}\right)}\left|u\left(t_{1}, s, x(\gamma(s))\right)\right| d_{s}
$$$$
\times\left(g\left(t_{2}, s\right)-g\left(t_{1}, s\right)\right)
$$$$
\leq \int_{\beta\left(t_{1}\right)}^{\beta\left(t_{2}\right)} k\left(t_{2}, s\right) \varphi(|x(\gamma(s))|) d_{s} g\left(t_{2}, s\right)
$$$$
+\int_{0}^{\beta\left(t_{1}\right)} \omega_{r_{0}}^{T}(u, \varepsilon) d_{s} g\left(t_{2}, s\right)
$$$$
+\int_{0}^{\beta\left(t_{1}\right)} k\left(t_{1}, s\right) \varphi(|x(\gamma(s))|) d_{s}
$$$$
\times\left(g\left(t_{2}, s\right)-g\left(t_{1}, s\right)\right)
$$$$
\leq k_{T} \varphi\left(r_{0}\right) \int_{\beta\left(t_{1}\right)}^{\beta\left(t_{2}\right)} d_{s} g\left(t_{2}, s\right)
$$$$
+\omega_{r_{0}}^{T}(u, \varepsilon) \int_{0}^{\beta\left(t_{1}\right)} d_{s} g\left(t_{2}, s\right)
$$$$
+k_{T} \varphi\left(r_{0}\right) \int_{0}^{\beta\left(t_{1}\right)} d_{s}\left(g\left(t_{2}, s\right)-g\left(t_{1}, s\right)\right)
$$$$
=k_{T} \varphi\left(r_{0}\right)\left[g\left(t_{2}, \beta\left(t_{2}\right)\right)-g\left(t_{2}, \beta\left(t_{1}\right)\right)\right]
$$$$
+\omega_{r_{0}}^{T}(u, \varepsilon)\left[g\left(t_{2}, \beta\left(t_{1}\right)\right)-g\left(t_{2}, 0\right)\right]
$$$$
+k_{T} \varphi\left(r_{0}\right)\left(\left[g\left(t_{2}, \beta\left(t_{1}\right)\right)-g\left(t_{1}, \beta\left(t_{1}\right)\right)\right]\right.
$$$$
\left.-\left[g\left(t_{2}, 0\right)-g\left(t_{1}, 0\right)\right]\right)
$$$$
\leq k_{T} \varphi\left(r_{0}\right)\left[g\left(T, \beta\left(t_{2}\right)\right)-g\left(T, \beta\left(t_{1}\right)\right)\right]
$$$$
+\omega_{r_{0}}^{T}(u, \varepsilon)\left[g\left(T, \beta\left(t_{1}\right)\right)-g(T, 0)\right]
$$$$
+k_{T} \varphi\left(r_{0}\right)\left(\left[g\left(t_{2}, \beta^{T}\right)-g\left(t_{1}, \beta^{T}\right)\right]\right.
$$$$
\left.+\left|g\left(t_{2}, 0\right)-g\left(t_{1}, 0\right)\right|\right)
$$$$
\leq k_{T} \varphi\left(r_{0}\right) \omega^{T}\left(g(T, \cdot), \omega^{T}(\beta, \varepsilon)\right)
$$$$
+\omega_{r_{0}}^{T}(u, \varepsilon)\left[g\left(T, \beta^{T}\right)-g(T, 0)\right]
$$$$
+k_{T} \varphi\left(r_{0}\right)\left[\omega^{T}\left(g\left(\cdot, \beta^{T}\right), \varepsilon\right)\right.
$$$$
\left.+\omega^{T}(g(\cdot, 0), \varepsilon)\right],
$$

where

$$
\begin{gathered}
\omega^{T}\left(g(T, \cdot), \omega^{T}(\beta, \varepsilon)\right) \\
=\sup \left\{\left|g\left(T, \theta_{2}\right)-g\left(T, \theta_{1}\right)\right|: \theta_{1}, \theta_{2} \in\left[0, \beta^{T}\right],\right. \\
\left.\quad\left|\theta_{2}-\theta_{1}\right|<\omega^{T}(\beta, \varepsilon)\right\},
\end{gathered}
$$




$$
\begin{aligned}
& \omega_{r_{0}}^{T}(u, \varepsilon) \\
& =\sup \left\{\left|u\left(t_{2}, s, x\right)-u\left(t_{1}, s, x\right)\right|: t_{1}, t_{2} \in[0, T],\right. \\
& \left.s \in\left[0, \beta^{T}\right],\left|t_{2}-t_{1}\right| \leq \varepsilon, x \in\left[-r_{0}, r_{0}\right]\right\} .
\end{aligned}
$$

By $\left(H_{4}\right)$, one has

$$
\begin{aligned}
\vartheta_{2}= & \mid \int_{0}^{\infty} v\left(t_{2}, s, x(\delta(s))\right) d s \\
& -\int_{0}^{\infty} v\left(t_{1}, s, x(\delta(s))\right) d s \mid \\
\leq & \int_{0}^{\infty} \mid v\left(t_{2}, s, x(\delta(s))\right) d s \\
\leq & \int_{0}^{T} \mid v\left(t_{2}, s, x(\delta(s))\right) d s \\
& +\int_{T}^{\infty}\left|v\left(t_{2}, s, x(\delta(s))\right) d s-v\left(t_{1}, s, x(\delta(s))\right)\right| d s \\
\leq & T \omega_{r_{0}}^{T}(v, \varepsilon)+\int_{T}^{\infty}\left|v\left(t_{2}, s, x(\delta(s))\right)\right| d s \\
& +\int_{T}^{\infty}\left|v\left(t_{1}, s, x(\delta(s))\right)\right| d s \\
\leq & T \omega_{r_{0}}^{T}(v, \varepsilon)+\int_{T}^{\infty} p\left(t_{2}, s\right) \psi(|x(\delta(s))|) d s \\
& +\int_{T}^{\infty} p\left(t_{1}, s\right) \psi(|x(\delta(s))|) d s \\
\leq & T \omega_{r_{0}}^{T}(v, \varepsilon) \\
+ & s u p\left\{\int_{T}^{\infty} p(t, s) d s: t \in \mathbb{R}^{+}\right\} \cdot \psi\left(r_{0}\right),
\end{aligned}
$$

where

$$
\begin{aligned}
& \omega_{r_{0}}^{T}(v, \varepsilon) \\
& =\sup \left\{\left|v\left(t_{2}, s, x\right)-v\left(t_{1}, s, x\right)\right|: t_{1}, t_{2}, \in[0, T],\right. \\
& \left.\quad s \in[0, T],\left|t_{2}-t_{1}\right| \leq \varepsilon, x \in\left[-r_{0}, r_{0}\right]\right\} .
\end{aligned}
$$

By (25), (27), (29), and (31), one has

$$
\begin{aligned}
\omega^{T}(\mathscr{F} x, \varepsilon) \leq & M \lambda \omega^{T}\left(x, \omega^{T}(\alpha, \varepsilon)\right)+M \omega_{r_{0}}^{T}(f, \varepsilon) \\
& +m_{1}^{T} k_{T} \varphi\left(r_{0}\right) \omega^{T}\left(g(T, \cdot), \omega^{T}(\beta, \varepsilon)\right) \\
& +m_{1}^{T} \omega_{r_{0}}^{T}(u, \varepsilon)\left[g\left(T, \beta^{T}\right)-g(T, 0)\right] \\
& +m_{1}^{T} k_{T} \varphi\left(r_{0}\right)\left[\omega^{T}\left(g\left(\cdot, \beta^{T}\right), \varepsilon\right)\right. \\
& \left.+\omega^{T}(g(\cdot, 0), \varepsilon)\right] \\
& +m_{2}^{T} T \omega_{r_{0}}^{T}(v, \varepsilon)+2 m_{2}^{T} \psi\left(r_{0}\right) \\
& \times \sup \left\{\int_{T}^{\infty} p(t, s) d s: t \in \mathbb{R}^{+}\right\}+\omega_{r_{0}}^{T}(F, \varepsilon),
\end{aligned}
$$

and then

$$
\begin{aligned}
\omega^{T}(\mathscr{F} X, \varepsilon) \leq & M \lambda \omega^{T}\left(X, \omega^{T}(\alpha, \varepsilon)\right) \\
& +M \omega_{r_{0}}^{T}(f, \varepsilon)+m_{1}^{T} k_{T} \varphi\left(r_{0}\right) \omega^{T} \\
& \times\left(g(T, \cdot), \omega^{T}(\beta, \varepsilon)\right) \\
& +m_{1}^{T} \omega_{r_{0}}^{T}(u, \varepsilon)\left[g\left(T, \beta^{T}\right)-g(T, 0)\right] \\
& +m_{1}^{T} k_{T} \varphi\left(r_{0}\right) \\
& \times\left[\omega^{T}\left(g\left(\cdot, \beta^{T}\right), \varepsilon\right)+\omega^{T}(g(\cdot, 0), \varepsilon)\right] \\
& +m_{2}^{T} T \omega_{r_{0}}^{T}(v, \varepsilon)+2 m_{2}^{T} \psi\left(r_{0}\right) \\
& \times \sup \left\{\int_{T}^{\infty} p(t, s) d s: t \in \mathbb{R}^{+}\right\}+\omega_{r_{0}}^{T}(F, \varepsilon) .
\end{aligned}
$$

By the uniform continuity of the functions $f, g, \beta, u, v$, and $F$ on compact set, one has

$$
\begin{gathered}
\omega_{r_{0}}^{T}(f, \varepsilon) \longrightarrow 0, \quad \omega_{r_{0}}^{T}(u, \varepsilon) \longrightarrow 0, \\
\omega_{r_{0}}^{T}(v, \varepsilon) \longrightarrow 0, \quad \omega_{r_{0}}^{T}(F, \varepsilon) \longrightarrow 0, \quad \text { as } \varepsilon \longrightarrow 0, \\
\omega^{T}\left(g(T, \cdot), \omega^{T}(\beta, \varepsilon)\right) \longrightarrow 0, \\
\omega^{T}\left(g\left(\cdot, \beta^{T}\right), \varepsilon\right) \longrightarrow 0, \quad \omega^{T}(g(\cdot, 0), \varepsilon) \longrightarrow 0, \quad \text { as } \varepsilon \longrightarrow 0,
\end{gathered}
$$

so

$$
\begin{aligned}
\omega_{0}^{T}(\mathscr{F} X) \leq & M \lambda \omega_{0}^{T}(X)+2 m_{2}^{T} \psi\left(r_{0}\right) \\
& \times \sup \left\{\int_{T}^{\infty} p(t, s) d s: t \in \mathbb{R}^{+}\right\},
\end{aligned}
$$

and then, by $\left(H_{8}\right)$,

$$
\omega_{0}(\mathscr{F} X) \leq M \lambda \omega_{0}(X) .
$$

On the other hand, take arbitrary $x, y \in X$ and fix $t \in \mathbb{R}^{+}$; one has

$$
\begin{aligned}
& \mid(\mathscr{F} x)(t)-(\mathscr{F} y)(t) \mid \\
& \leq M|f(t, x(\alpha(t)))-f(t, y(\alpha(t)))|+m_{1}(t) \\
& \times\left|\int_{0}^{\beta(t)}[u(t, s, x(\gamma(s)))-u(t, s, y(\gamma(s)))] d_{s} g(t, s)\right| \\
&+m_{2}(t)\left|\int_{0}^{\infty}[v(t, s, x(\delta(s)))-v(t, s, y(\delta(s)))] d s\right| \\
& \leq M \lambda|x(\alpha(t))-y(\alpha(t))|+m_{1}(t) \\
& \quad \times \int_{0}^{\beta(t)}|u(t, s, x(\gamma(s)))-u(t, s, y(\gamma(s)))| d_{s} \\
& \quad \times\left(\bigvee_{p=0}^{s} g(t, p)\right) \\
& \quad+m_{2}(t) \int_{0}^{\infty}|v(t, s, x(\delta(s)))-v(t, s, y(\delta(s)))| d s
\end{aligned}
$$




$$
\begin{aligned}
& \leq M \lambda \operatorname{diam} X(t)+m_{1}(t) \\
& \quad \times \int_{0}^{\beta(t)} k(t, s) \varphi(|x(\gamma(s))|) d_{s} g(t, s) \\
& \quad+m_{1}(t) \int_{0}^{\beta(t)} k(t, s) \varphi(|y(\gamma(s))|) d_{s} g(t, s) \\
& +m_{2}(t) \int_{0}^{\infty} p(t, s) \psi(|x(\delta(s))|) d s \\
& \quad+m_{2}(t) \int_{0}^{\infty} p(t, s) \psi(|y(\delta(s))|) d s \\
& \leq \\
& M \lambda \operatorname{diam} X(t)+2 \varphi\left(r_{0}\right) m_{1}(t) \\
& \quad \times \int_{0}^{\beta(t)} k(t, s) d_{s} g(t, s) \\
& \quad+2 \psi\left(r_{0}\right) m_{2}(t) \int_{0}^{\infty} p(t, s) d s,
\end{aligned}
$$

and hence

$$
\begin{aligned}
\operatorname{diam}(\mathscr{F} X)(t) \leq & M \lambda \operatorname{diam} X(t) \\
& +2 \varphi\left(r_{0}\right) m_{1}(t) \\
& \times \int_{0}^{\beta(t)} k(t, s) d_{s} g(t, s) \\
& +2 \psi\left(r_{0}\right) m_{2}(t) \int_{0}^{\infty} p(t, s) d s .
\end{aligned}
$$

By $\left(H_{7}\right)$, one has

$$
\limsup _{t \rightarrow \infty} \operatorname{diam}(\mathscr{F} X)(t) \leq M \lambda \limsup _{t \rightarrow \infty} \operatorname{diam} X(t) .
$$

By (37) and (40), one has

$$
\mu(\mathscr{F} X) \leq M \lambda \mu(X),
$$

where $\mu$ is the measure of noncompactness defined in (7). Hence, in view of Theorem 7 , we conclude that $\mathscr{F}$ has at least one fixed-point $x \in B_{r_{0}}$, which is a solution of (1). Moreover, taking into account the description of sets belonging to ker $\mu$ (see Remark 4), we deduce that the solutions of (1) are globally asymptotically stable. This completes the proof.

Remark 13. Equation (1) is quite general and includes some nonlinear integral equations studied earlier by various authors as special cases. For example, we have the following.

(i) If $f(t, x)=x, g(t, s)=s, \alpha(t)=\beta(t)=\gamma(t)=\delta(t)=$ $t$, (1) reduces to the nonlinear functional integral equation which studied by [7], that is,

$$
\begin{aligned}
& x(t)=F\left(t, x(t), \int_{0}^{t} u(t, s, x(s)) d s,\right. \\
& \left.\qquad \int_{0}^{\infty} v(t, s, x(s)) d s\right), \quad t \in \mathbb{R}^{+} .
\end{aligned}
$$

(ii) If $F(t, x, y, z)=q(t)+x y, \alpha(t)=\beta(t)=\gamma(t)=\delta(t)=$ $t, v(t, s, x)=0$, (1) reduces to the nonlinear quadratic integral equation of Volterra-Stieltjes type which is studied by the author of [2], that is,

$$
\begin{aligned}
x(t)= & q(t)+f(t, x(t)) \\
& \times \int_{0}^{t} u(t, s, x(s)) d_{s} g(t, s), \quad t \in \mathbb{R}^{+} .
\end{aligned}
$$

(iii) If $F(t, x, y, z)=q(t)+x z, g(t, s)=s, \alpha(t)=\beta(t)=$ $\gamma(t)=\delta(t)=t, u(t, s, x)=0$, (1) reduces to the quadratic Urysohn integral equation which is studied by the author of [22], that is,

$$
\begin{aligned}
x(t)= & q(t)+f(t, x(t)) \\
& \times \int_{0}^{\infty} v(t, s, x(s)) d s, \quad t \in \mathbb{R}^{+} .
\end{aligned}
$$

For more detail, one can see Table 1.

\section{Examples}

In this section, we present some examples to illustrate our main result.

Example 14. Consider the nonlinear functional integral equation of Volterra-Stieltjes type

$$
\begin{aligned}
x(t)= & f(t, x(\alpha(t))) \\
& +\int_{0}^{\beta(t)} u(t, s, x(\gamma(s))) d_{s} g(t, s), \quad t \in \mathbb{R}^{+} .
\end{aligned}
$$

Equation (45) is a special case of (1), where $F(t, x, y, z)=$ $x+y, v(t, s, x)=0, \delta(t)=0$. Since $F(t, x, y, z)=x+y,\left(H_{5}\right)$ is satisfied with $M=m_{1}(t)=1, m_{2}(t)=0$, and $F_{0}=0$. By Theorem 12, one has the following.

Theorem 15. Assume that $\left(H_{1}\right)-\left(H_{3}\right),\left(H_{6}\right),\left(H_{7}\right)$, and $\left(H_{9}\right)$ hold; then (45) has at least one solution in $B C\left(\mathbb{R}^{+}\right)$which is globally asymptotically stable.

Remark 16. When $g(t, s)=s,(45)$ is investigated by [3], and if $g(t, s)=s, \alpha(t)=\beta(t)=\gamma(t)=t$, it is investigated by the author of [6], see Table 1 for more details. Theorem 15 improves, and generalizes the results of $[3,6]$.

Remark 17. If $F(t, x, y, z)=q(t)+x y$, the following quadratic nonlinear functional integral equation of Volterra-Stieltjes type:

$$
\begin{aligned}
x(t)= & q(t)+f(t, x(\alpha(t))) \\
& \times \int_{0}^{\beta(t)} u(t, s, x(\gamma(s))) d_{s} g(t, s), \quad t \in \mathbb{R}^{+} .
\end{aligned}
$$

When $\alpha(t)=\beta(t)=\gamma(t)=t$ in (46), it is investigated by the authors of [2]. For (46), by Theorem 12, one has a similar result. Here we omit it. 
TABLE 1: Some of papers studied earlier as special cases, where $q, \psi, \varphi, k, h$ are suitable continuous functions.

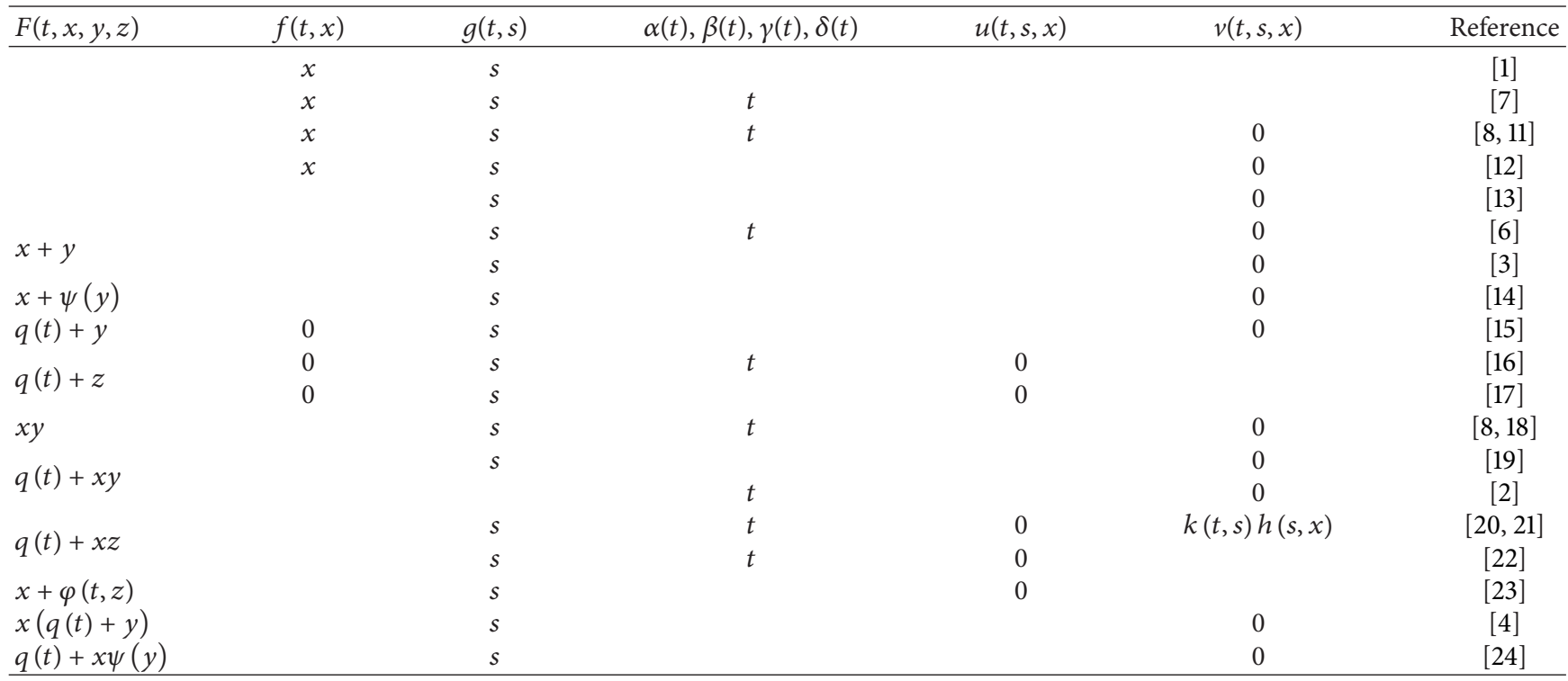

Example 18. Consider the quadratic Urysohn functional integral equation

$$
\begin{aligned}
x(t)= & q(t)+f(t, x(\alpha(t))) \\
& \times \int_{0}^{\infty} v(t, s, x(\delta(s))) d s \quad t \in \mathbb{R}^{+} .
\end{aligned}
$$

Equation (47) is a special case of (1), where $F(t, x, y, z)=$ $q(t)+x z, u(t, s, x)=0$, and $\beta(t)=\gamma(t)=0$. By Theorem 12, one has the following theorem.

Theorem 19. Assume that $\left(H_{1}\right)$ and $\left(H_{4}\right)-\left(H_{9}\right)$ hold, and (47) has at least one solution in $B C\left(\mathbb{R}^{+}\right)$which is globally asymptotically stable.

Finally, we provide an example of concrete nonlinear functional integral equation of (1) which satisfies all the assumptions of Theorem 12 .

Example 20. Consider the functional integral equation

$$
\begin{aligned}
x(t)= & \frac{1}{32} e^{-t^{2}}+\frac{t^{2}+2}{32\left(t^{2}+1\right)} x(2 t) \\
& +\frac{1}{1+t^{2}} \int_{0}^{t^{2}} \frac{x^{2}(s)}{1+t^{4}+s^{2}} d s \\
& +\frac{1}{4} \sin ^{2} t \int_{0}^{\infty} x(s) e^{-s e^{t}} d s, \quad t \in \mathbb{R}^{+} .
\end{aligned}
$$

Observe that (48) is a special case of (1), where $F(t, x, y, z)=x+\left(1 /\left(1+t^{2}\right)\right) y+z \sin ^{2} t, f(t, x)=(1 / 32) e^{-t^{2}}+$ $\left(\left(t^{2}+2\right) /\left(32\left(t^{2}+1\right)\right)\right) x, u(t, s, x)=x^{2} /\left(1+t^{4}+s^{2}\right), v(t, s, x)=$ $(1 / 4) x e^{-s e^{t}}, g(t, s)=s, \alpha(t)=2 t, \beta(t)=t^{2}$, and $\gamma(t)=\delta(t)=$ $t$. Then

$$
\begin{gathered}
|u(t, s, x)| \leq \frac{1}{1+t^{4}+s^{2}} x^{2}, \\
|v(t, s, x)-v(t, s, y)| \leq \frac{1}{4} e^{-s e^{t}}|x-y|,
\end{gathered}
$$

so $k(t, s)=1 /\left(1+t^{4}+s^{2}\right), p(t, s)=(1 / 4) e^{-s e^{t}}, \varphi(x)=x^{2}$, and $\psi(x)=x$, and then $\left(H_{1}\right)-\left(H_{4}\right)$ hold.

By $F(t, x, y, z)=x+\left(1 /\left(1+t^{2}\right)\right) y+z \sin ^{2} t$ and $f(t, x)=$ $(1 / 32) e^{-t^{2}}+\left(\left(t^{2}+2\right) /\left(32\left(t^{2}+1\right)\right)\right) x$, it is easy to see that $\left(H_{5}\right)$ and $\left(H_{6}\right)$ are satisfied with $M=1, m_{1}(t)=1 /\left(1+t^{2}\right), m_{2}(t)=$ $\sin ^{2} t, \lambda=1 / 16$, and $F_{0}=0, f_{0}=1 / 32$. Since

$$
\begin{aligned}
& \frac{1}{1+t^{4}+s^{2}} \leq \frac{1}{1+s^{2}} \\
& \int_{0}^{\infty} p(t, s) d s=\frac{1}{4} e^{-t}
\end{aligned}
$$

which implies that

$$
\begin{gathered}
\lim _{t \rightarrow \infty} \int_{0}^{\beta(t)} k(t, s) d_{s} g(t, s) \leq \lim _{t \rightarrow \infty} \int_{0}^{t^{2}} \frac{1}{1+s^{2}} d s=\frac{\pi}{2}, \\
\lim _{t \rightarrow \infty} \int_{0}^{\infty} p(t, s) d s=0,
\end{gathered}
$$

so $\left(H_{7}\right)$ holds. It can be easily seen that $p(t, s)$ satisfies $\left(H_{8}\right)$. Moreover,

$$
\begin{aligned}
M_{1}= & \sup \left\{\frac{1}{1+t^{2}} \int_{0}^{t^{2}} \frac{1}{1+t^{4}+s^{2}} d s: t \geq 0\right\} \leq \frac{\pi}{2}, \\
M_{2} & =\sup \left\{\frac{1}{4} \sin ^{2} t \int_{0}^{\infty} e^{-s e^{t}} d s: t \geq 0\right\} \\
& =\sup \left\{\frac{1}{4} e^{-t} \sin ^{2} t: t \geq 0\right\} \leq \frac{1}{4},
\end{aligned}
$$

then the inequality from $\left(H_{9}\right)$ has the following form:

$$
\frac{1}{16} r+\frac{1}{32}+\frac{\pi}{2} r^{2}+\frac{1}{4} r \leq r .
$$

Obviously, $r_{0}=1 / 8$ is a positive solution of this inequality for which $M \lambda<1$, and thus $\left(H_{9}\right)$ holds. By Theorem 12, (48) has 
at least one solution in $\mathrm{BC}\left(\mathbb{R}^{+}\right)$which is globally asymptotically stable.

\section{Acknowledgment}

This paper is supported by Zhejiang Provincial Natural Science Foundation of China under Grant no. LQ13A010015.

\section{References}

[1] R. P. Agarwal, J. Banaś, B. C. Dhage, and S. D. Sarkate, "Attractivity results for a nonlinear functional integral equation," Georgian Mathematical Journal, vol. 18, no. 1, pp. 1-19, 2011.

[2] J. Banaś, "Existence results for Volterra-Stieltjes quadratic integral equations on an unbounded interval," Mathematica Scandinavica, vol. 98, no. 1, pp. 143-160, 2006.

[3] J. Banaś and B. C. Dhage, "Global asymptotic stability of solutions of a functional integral equation," Nonlinear Analysis. Theory, Methods \& Applications A, vol. 69, no. 7, pp. 1945-1952, 2008.

[4] B. C. Dhage, "Local asymptotic attractivity for nonlinear quadratic functional integral equations," Nonlinear Analysis. Theory, Methods \& Applications A, vol. 70, no. 5, pp. 1912-1922, 2009.

[5] A. Sikorska-Nowak, "Existence theory for integrodifferential equations and Henstock-Kurzweil integral in Banach spaces," Journal of Applied Mathematics, vol. 2007, Article ID 31572, 12 pages, 2007.

[6] J. Banaś and B. Rzepka, "An application of a measure of noncompactness in the study of asymptotic stability," Applied Mathematics Letters, vol. 16, no. 1, pp. 1-6, 2003.

[7] M. A. Darwish, "On global attractivity of solutions of a functional-integral equation," Electronic Journal of Qualitative Theory of Differential Equations, vol. 21, pp. 1-10, 2007.

[8] X. Hu and J. Yan, "The global attractivity and asymptotic stability of solution of a nonlinear integral equation," Journal of Mathematical Analysis and Applications, vol. 321, no. 1, pp. 147-156, 2006.

[9] J. Banaś and D. O’Regan, "Volterra-Stieltjes integral operators," Mathematical and Computer Modelling, vol. 41, no. 2-3, pp. 335344, 2005.

[10] J. Banaś and K. Goebel, Measures of Noncompactness in Banach Spaces, vol. 60, Marcel Dekker, New York, NY, USA, 1980.

[11] J. Banaś, J. Caballero, B. López, and K. Sadarangani, “Solutions of a functional integral equation in $B C\left(\mathbb{R}_{+}\right)$," International Mathematical Forum. Journal for Theory and Applications, vol. 1, no. 21-24, pp. 1181-1194, 2006.

[12] A. Aghajani and Y. Jalilian, "Existence and global attractivity of solutions of a nonlinear functional integral equation," Communications in Nonlinear Science and Numerical Simulation, vol. 15, no. 11, pp. 3306-3312, 2010.

[13] B. C. Dhage and V. Lakshmikantham, "On global existence and attractivity results for nonlinear functional integral equations," Nonlinear Analysis. Theory, Methods \& Applications A, vol. 72, no. 5, pp. 2219-2227, 2010.

[14] A. Aghajani and Y. Jalilian, "On the existence and global attractivity of solutions of a functional integral equation," International Journal of Computational and Mathematical Sciences, vol. 3, no. 7, pp. 347-353, 2009.

[15] D. O'Regan and M. Meehan, Existence Theory for Nonlinear Integral and Integrodifferential Equations, vol. 445 of Mathematics and its Applications, Kluwer Academic, Dordrecht, The Netherlands, 1998.

[16] J. Banaś and M. Pasławska-Południak, "Monotonic solutions of Urysohn integral equation on unbounded interval," Computers \& Mathematics with Applications, vol. 47, no. 12, pp. 1947-1954, 2004.

[17] A. A. El-Bary, M. A. Darwish, and W. G. El-Sayed, "On an existence theorem for Urysohn integral equations via measure of noncompactness," Mathematical Sciences Research Journal, vol. 6, no. 9, pp. 441-448, 2002.

[18] J. Banaś and B. Rzepka, "On existence and asymptotic stability of solutions of a nonlinear integral equation," Journal of Mathematical Analysis and Applications, vol. 284, no. 1, pp. 165-173, 2003.

[19] B. C. Dhage and S. S. Bellale, "Local asymototic stability for nonlinear quadratic functional integral equations," Electronic Journal of Qualitative Theory of Differential Equations, vol. 10, pp. 113,2008 .

[20] J. Banaś, J. Rocha Martin, and K. Sadarangani, "On solutions of a quadratic integral equation of Hammerstein type," Mathematical and Computer Modelling, vol. 43, no. 1-2, pp. 97-104, 2006.

[21] J. Banaś, D. O’Regan, and R. P. Agarwal, "Measures of noncompactness and asymptotic stability of solutions of a quadratic Hammerstein integral equation," The Rocky Mountain Journal of Mathematics, vol. 41, no. 6, pp. 1769-1792, 2011.

[22] J. Banaś and L. Olszowy, "On solutions of a quadratic Urysohn integral equation on an unbounded interval," Dynamic Systems and Applications, vol. 17, no. 2, pp. 255-269, 2008.

[23] M. A. Darwish, "On a perturbed functional integral equation of Urysohn type," Applied Mathematics and Computation, vol. 218, no. 17, pp. 8800-8805, 2012.

[24] A. Aghajani and N. Sabzali, "Existence and local attractivity of solutions of a nonlinear quadratic functional integral equation," Iranian Journal of Science and Technology A, vol. 36, no. 4, pp. 453-460, 2012. 


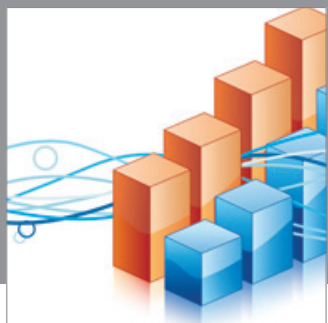

Advances in

Operations Research

mansans

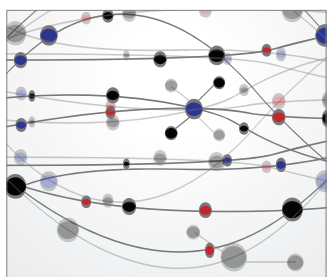

The Scientific World Journal
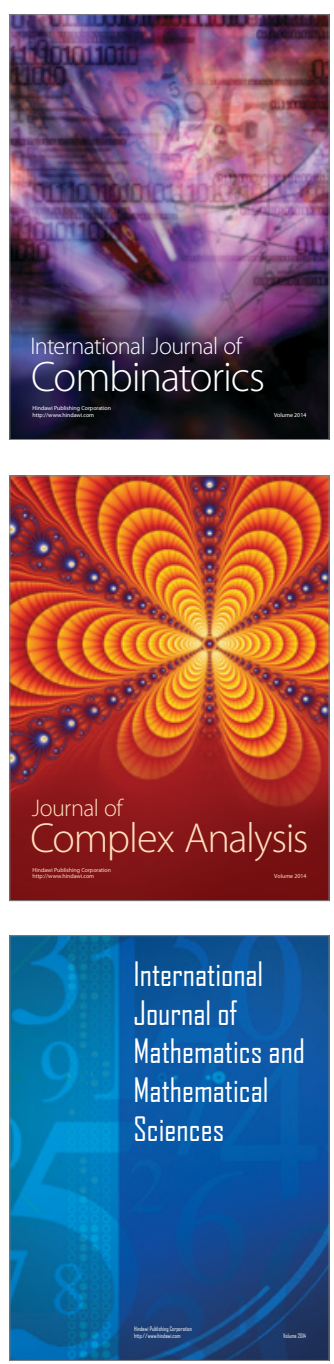
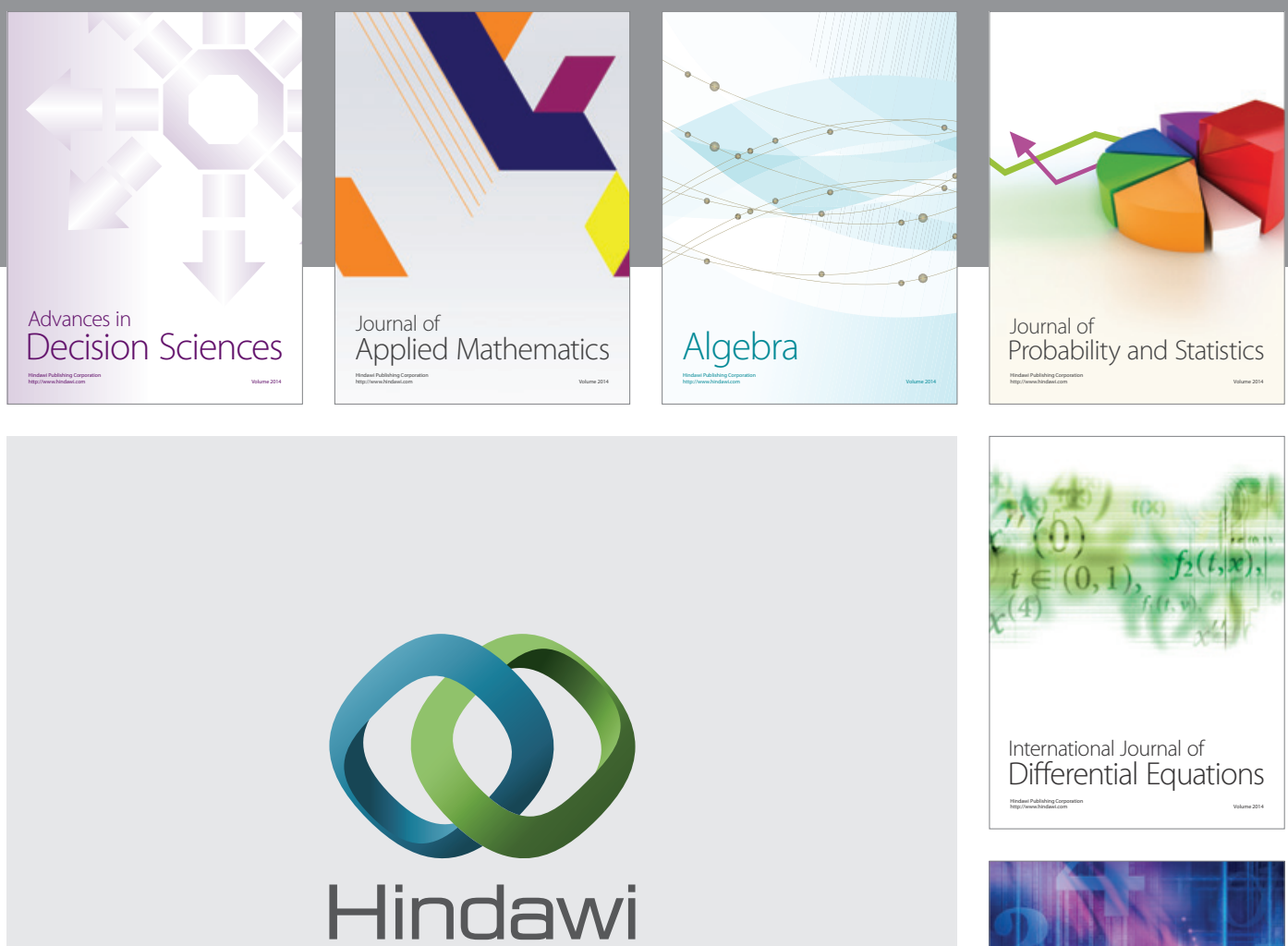

Submit your manuscripts at http://www.hindawi.com
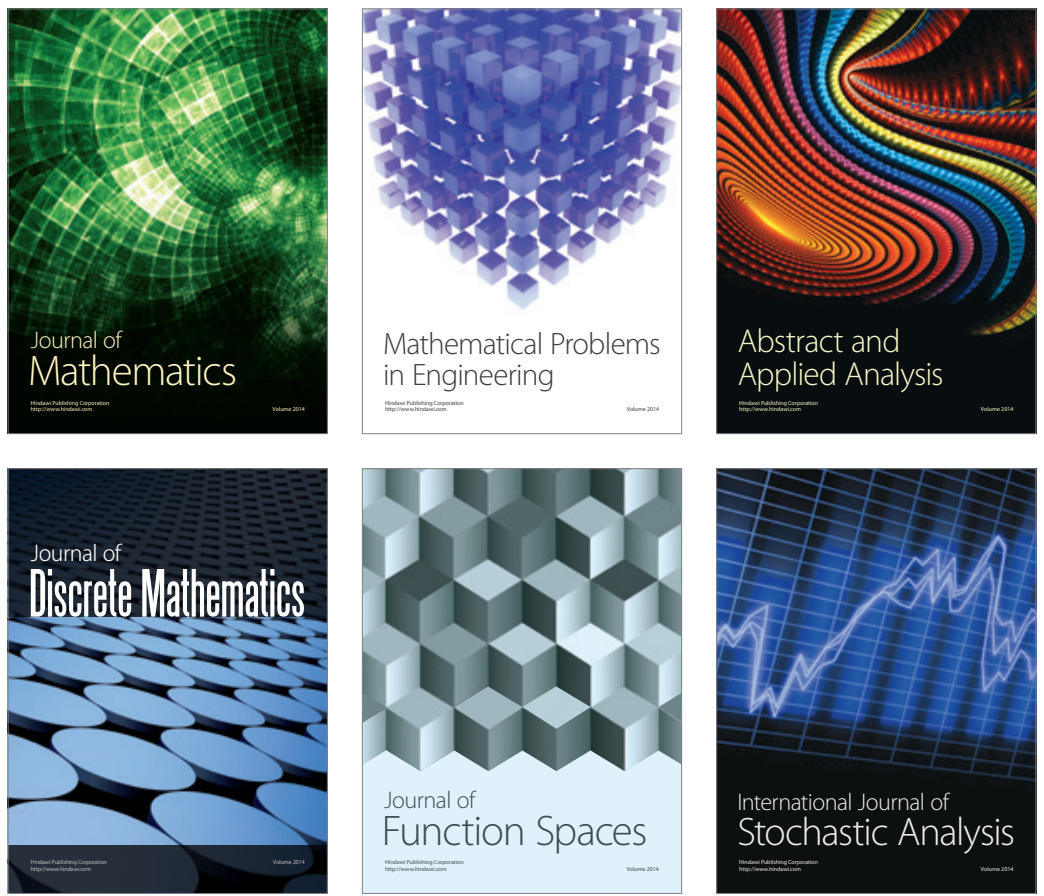

Journal of

Function Spaces

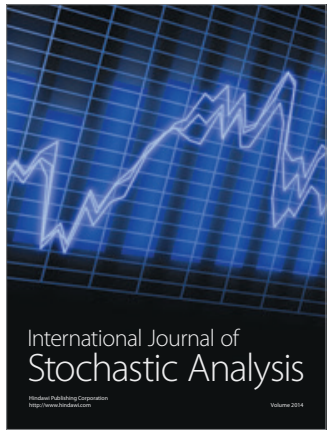

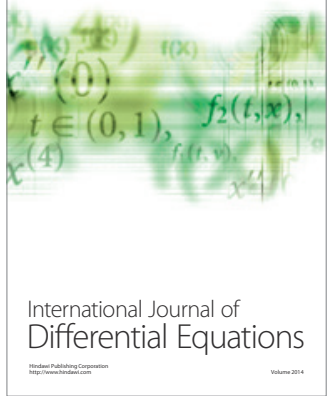
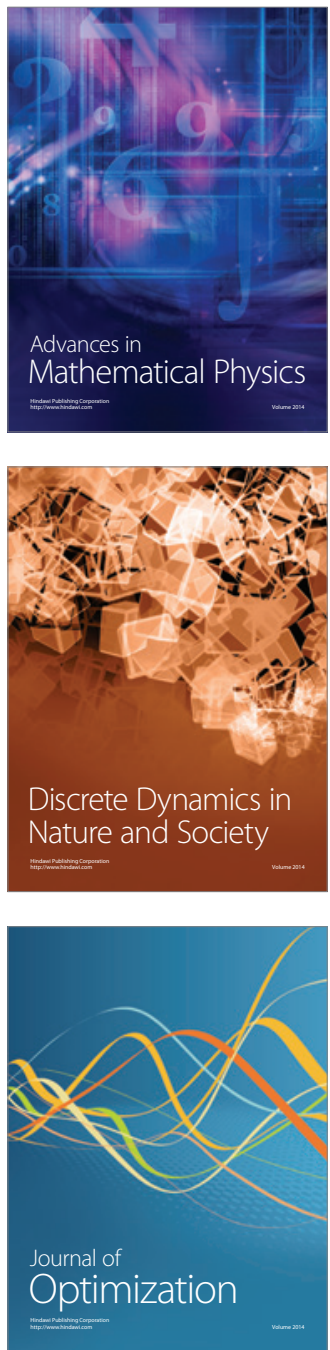